\title{
Recognition of microscopic colitis at colonoscopy
}

\author{
Marietta lacucci MD PhD¹, Stefan Urbanski MD²
}

$\mathrm{M}$ icroscopic colitis is a common condition in Canada and is characterized by watery diarrhea with normal endoscopic mucosal appearance using conventional white-light endoscopy. Two types of microscopic colitis are recognized by histological appearance: lymphocytic colitis and collagenous colitis. Recent studies suggest that $10 \%$ to $30 \%$ of older patients who present with diarrhea and demonstrate normal mucosal appearance at endoscopy have microscopic colitis (1). Increasingly, however, with high-definition endoscopy assisted by postprocessing of images by filter techniques in combination with chromoendoscopy, subtle abnormalities of the mucosa are apparent in patients with microscopic colitis.

iScan (Pentax, Japan) is a newly developed postprocessing light filter that enhances details of the mucosal surface, mucosal patterns (i-Scan $\mathrm{p}$ designated iScan 1) and vessel architecture (i-Scan v designated iScan 2). i-Scan is a technique designed for differentiating neoplastic from non-neoplastic lesions in the colon. This new digital modality can characterize mucosal patterns of the gastrointestinal mucosa in detail. Subtle abnormalities can, therefore, be recognized as either neoplastic or inflammatory.
Hoffman et al (2) have tested the efficacy of high-definition endoscopy alone in comparison with i-Scan or chromoendoscopy with methylene blue $(0.1 \%)$ in screening for colorectal cancer. They demonstrated that both i-Scan and chromoendoscopy identified more lesions compared with high-definition endoscopy alone. i-Scan was also able to predict neoplasia as precisely as chromoendoscopy, with an accuracy of $89 \%$ to $97 \%$.

\section{CASE PRESENTATION}

A 50-year-old woman with persistent undiagnosed chronic watery diarrhea underwent colonoscopy, with random biopsies that were negative. Another colonoscopy was performed with iScan + chromoendoscopy with $0.2 \%$ indigo carmine to enhance mucosal surfaces and patterns, and to target biopsies in the colon. iScan 1 and iScan 2 showed irregularity of the colonic mucosal pattern, although the mucosa appeared normal with white-light endoscopy. iScan + chromoendoscopy with indigo carmine characterized, in detail, the mucosal pattern as nodularmosaic with a small honeycomb pit-pattern appearance (Figure 1). Such an appearance is characteristic of microscopic colitis. Targeted
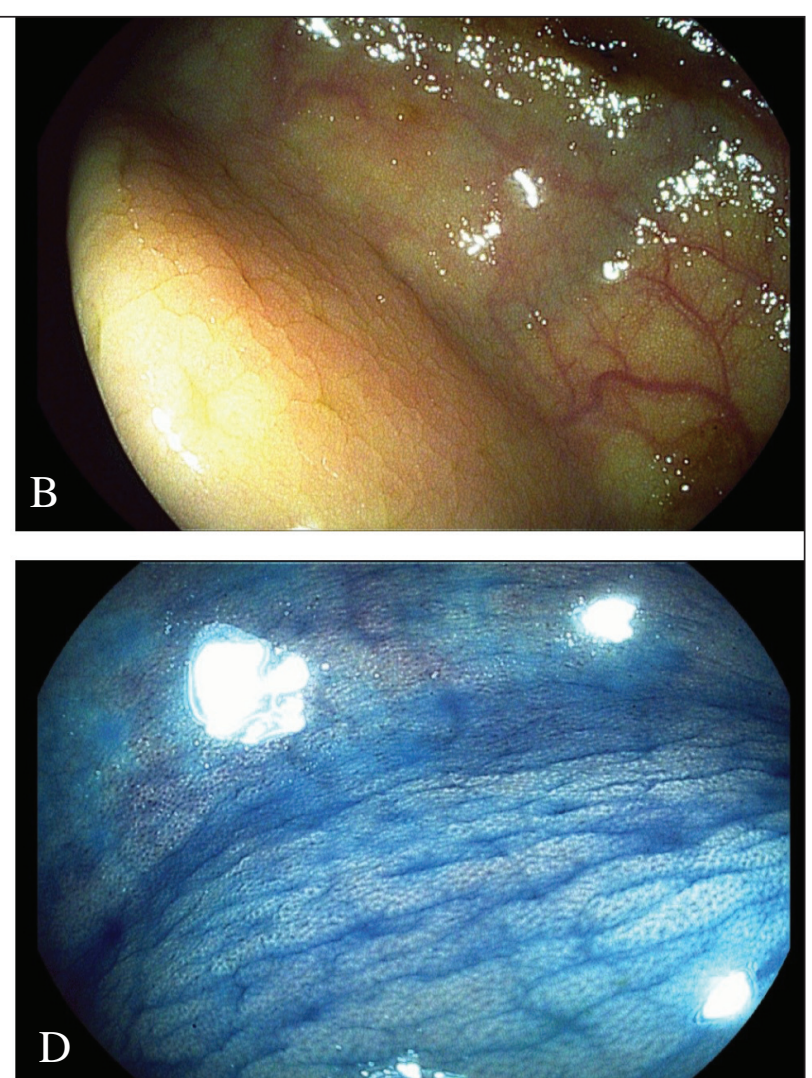

Figure 1) Endoscopic mucosal appearance of lymphocytic colitis with iScan 1and iScan 2 (Pentax, Japan) + chromoendoscopy with indigo carmine. A iScan 1 irregular mosaic pattern. B iScan 2 nodular mucosal pattern. C iScan $1+$ chromoendoscopy with 2\% indigo carmine showing a nodularmosaic pattern. D iScan2 + chromoendoscopy with indigo carmine showing a mosaic pattern with small honeycomb pit-pattern appearance

${ }^{1}$ Department of Medicine, Division of Gastroenterology; ${ }^{2}$ Department of Pathology, University of Calgary, Calgary, Alberta

Correspondence: Dr Marietta Iacucci, Division of Gastroenterology University of Calgary, Teaching, Research and Wellness Centre, 6D25,

3280 Hospital Drive Northwest, Calgary, Alberta T2N 4Z6. Telephone 403-592-5034, fax 403-592-5090, e-mail miacucci@ucalgary.ca

Received and accepted for publication December 14, 2011 

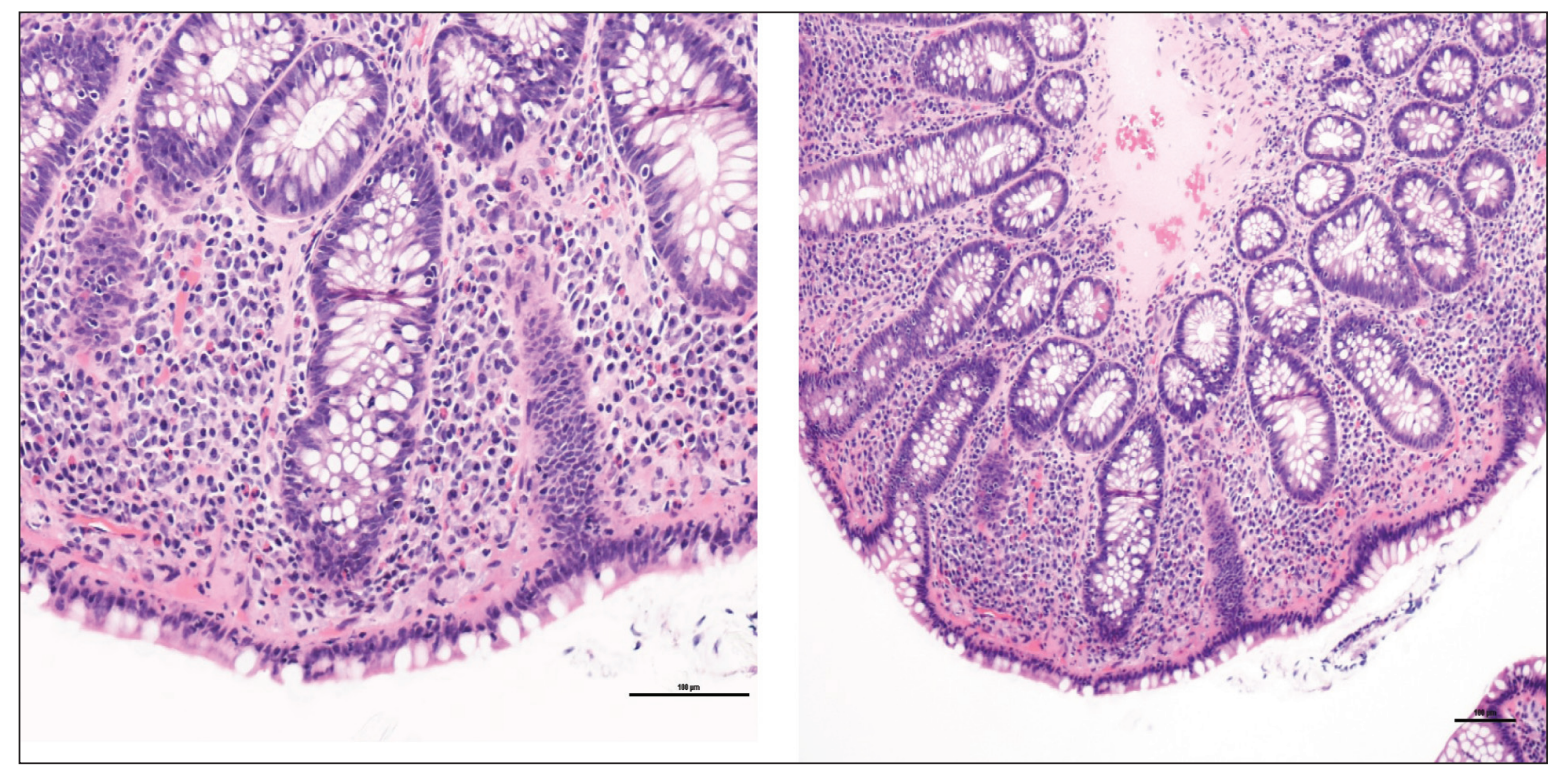

Figure 2) Hematoxylin and eosin stain showing increased intraepithelial lymphocytes. The lamina propria has an increased number of plasma cells and lymphocytes. Original magnification $\times 20$ (left panel); $\times 10$ (right panel)

biopsies were performed, and histology revealed lymphocytic colitis characterized by an increased number of intraepithelial lymphocytes and superficial infiltration of the lamina propria with inflammatory mononuclear cells (Figure 2).

\section{DISCUSSION}

Conventional white-light endoscopy classically describes microscopic colitis as having normal mucosal appearance with microscopic inflammation at histology. It may be limited in extent or patchy and, therefore, may be missed on random biopsies unless biopsies are numerous. The iScan technique, in combination with dye chromoendoscopy, might unmask endoscopic abnormalities of the colonic mucosa that are characteristic and recognizable. This could improve the diagnosis of microscopic colitis by targeting biopsies and prompting more extensive biopsies. For the first time, iScan with chromoendoscopy may permit macroscopic endoscopic recognition of microscopic colitis. Further studies on the sensitivity and specificity of this approach are required.

Suzuki et al (3) reported that chromoendoscopy using indigo carmine dye spray at colonoscopy can improve detection and characterization of lymphocytic and collagenous colitis. They recognized that a mosaic mucosal pattern represents lymphocytic colitis, while a nodulargrooved mucosal pattern was observed more often in collagenous colitis. However, these appearances may overlap with one another (3). Cimmino et al (4) have also confirmed that the findings of a mosaic pattern might be associated with microscopic colitis. Therefore, subtle inflammation previously recognized only by histology can now be identified by a new generation of high-definition and high-resolution endoscopic equipment combined with filter enhancement techniques such as iScan combined with chromoendoscopy. This may also be relevant for describing mucosal healing in patients with inflammatory bowel disease, which is currently a topic of great interest (5).

DISCLOSURES: The authors have no financial disclosures or conflicts of interest to declare.

\section{REFERENCES}

1. Williams JJ, Beck PL, Andrews CN, Hogan DB, Storr MA.

Microscopic colitis - a common cause of diarrhea in older adults.

Age Ageing 2010;39:162-8

2. Hoffman A, Kagel C, Goetz M, et al. Recognition and characterization of small colonic neoplasia with i-SCAN definition colonoscopy using iScan is as precise as chromoendosocpy. Dig Liver Dis 2010:42;45-5.

3. Suzuki G, Meliander MR, Suzuki A, et al The usefulness of colonoscopy examination with indigo carmine in diagnosing microscopic colitis. Endoscopy 2011;43:1100-4.

4. Cimmino DG, Mella JM, Pereyra L. A colorectal mosaic pattern might be an endoscopic feature of collagenous colitis. J Crohns Colitis 2010;4:139-43.

5. Iacucci M, Ghosh S. Looking beyond symptoms relief: Evolution of mucosal healing in inflammatory bowel disease. Therap Adv Gastroenterol 2011;4:129-43.

The Canadian Journal of Gastroenterology is now considering a limited number of submissions for IMAGE OF THE MONTH. These are based on endoscopic, histological, radiological and/or patient images, which must be anonymous with no identifying features visible. The patient must consent to publication and the consent must be submitted with the manuscript. All manuscripts should be practical and relevant to clinical practice, and not simply a case report of an esoteric condition. The text should be brief, structured as CASE PRESENTATION and DISCUSSION, and not more than 700 words in length. A maximum of three images can be submitted and the number of references should not exceed five. The submission may be edited by our editorial team.

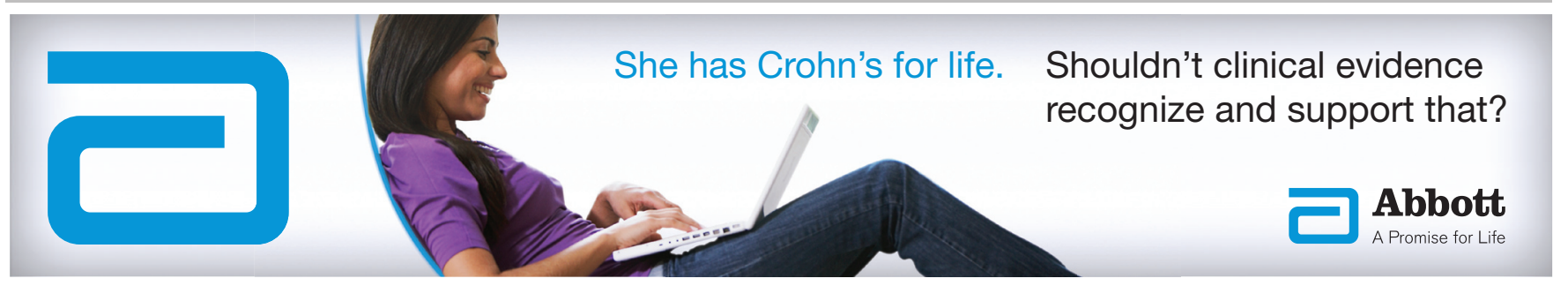




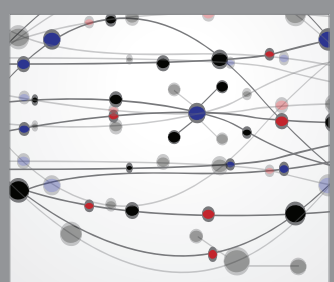

The Scientific World Journal
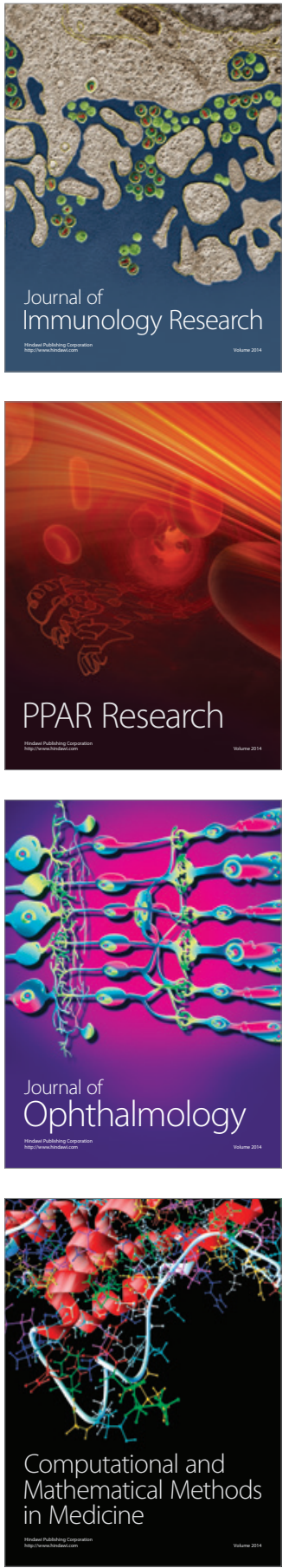

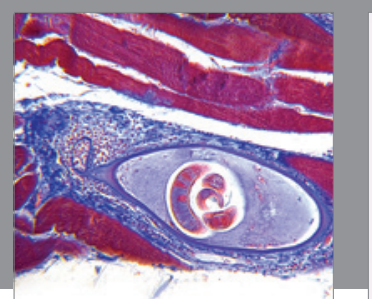

Gastroenterology Research and Practice

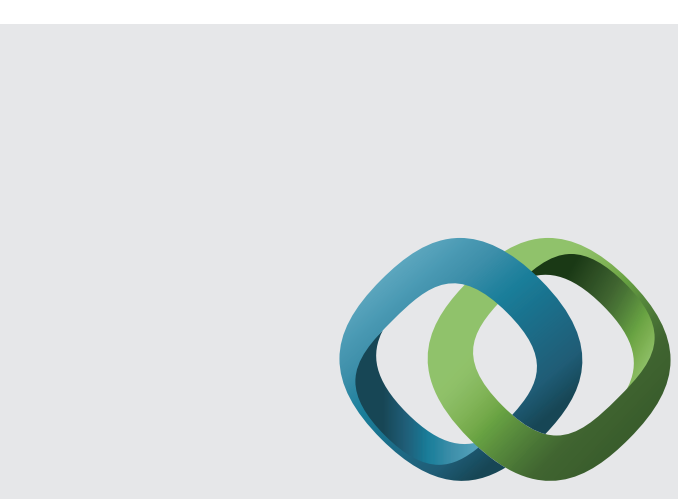

\section{Hindawi}

Submit your manuscripts at

http://www.hindawi.com
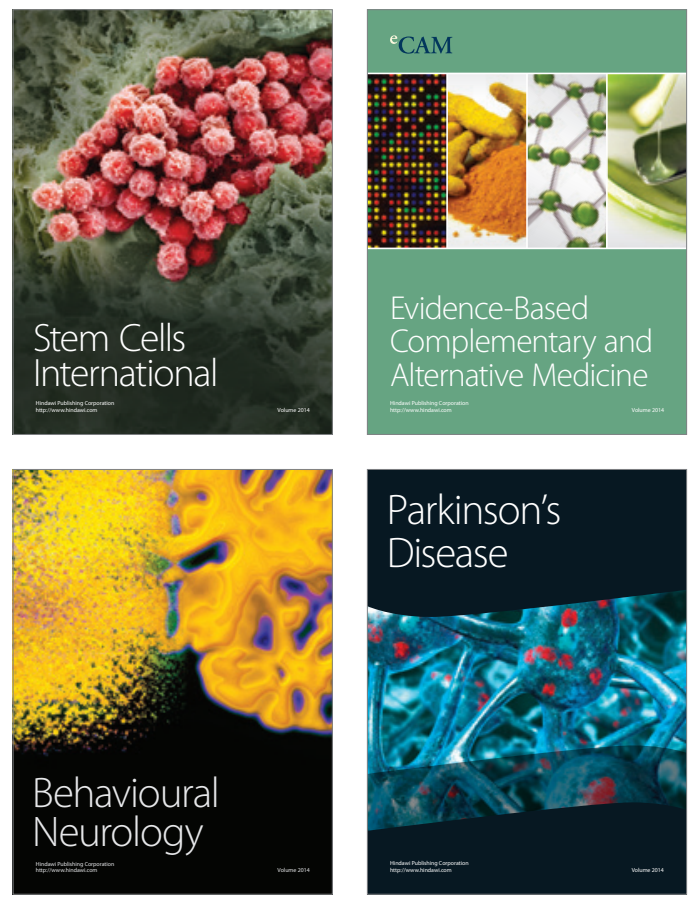
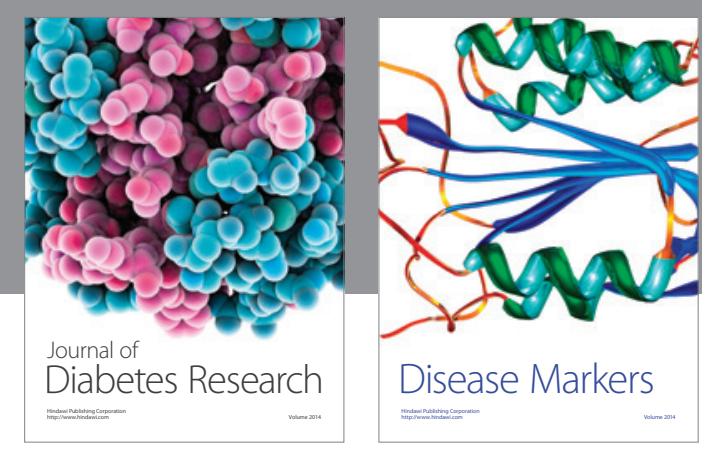

Disease Markers
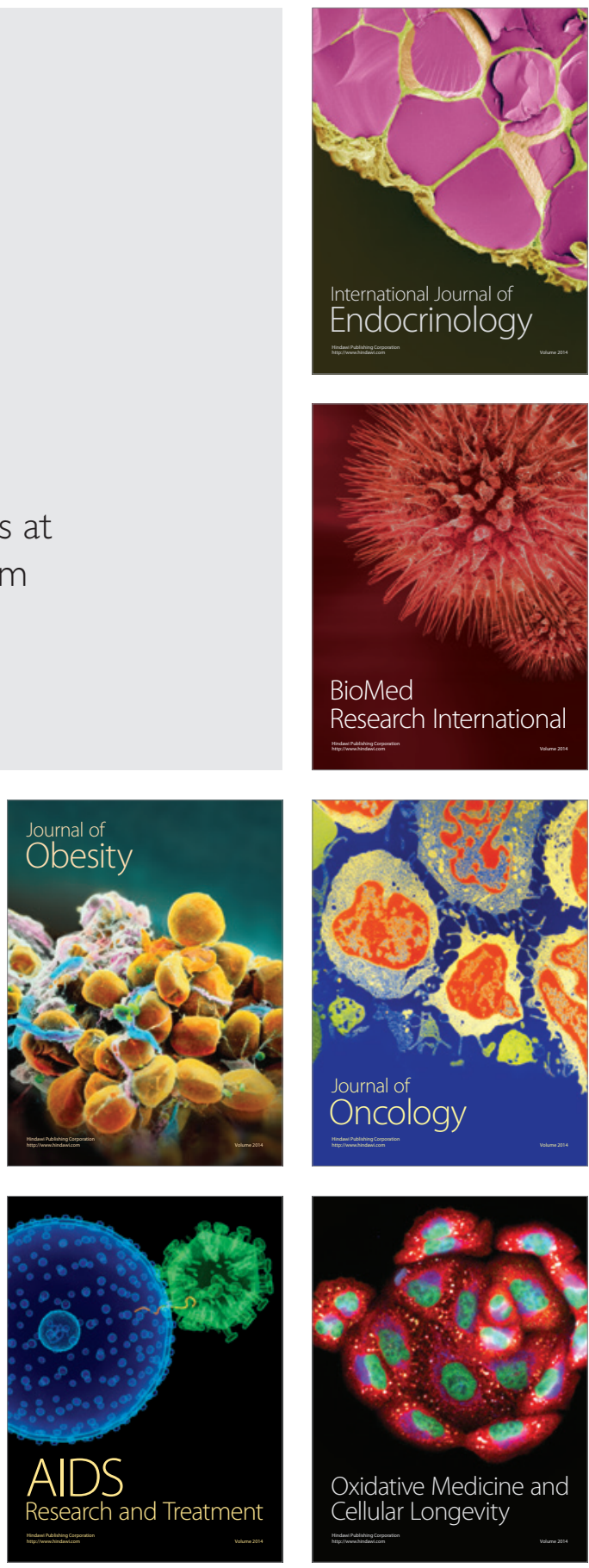